\title{
Cultura de segurança em uma maternidade pública de ensino: conhecer para intervir
}

\section{Safety culture in a public teaching maternity: knowing to intervene}

Cláudia Cecília Hernandez Barillas ${ }^{1}$. Ana Cláudia de Brito Passos ${ }^{1}$. Carlos Augusto Alencar Júnior ${ }^{1,2}$. Emeline Moura Lopes ${ }^{1,2}$. Eugenie Desiree Rabelo Neri ${ }^{1,2}$. Marta Maria de França Fonteles ${ }^{1}$. Djanilson Barbosa dos Santos ${ }^{1}$.

1 Universidade Federal do Ceará (UFC), Fortaleza, Ceará, Brasil. 2 Maternidade Escola Assis Chateaubriand (MEAC), Fortaleza, Ceará, Brasil. 3 Universidade Federal do Recôncavo da Bahia (UFRB), Cruz das Almas, Bahia, Brasil.

\section{RESUMO}

Objetivo: Avaliar a cultura de segurança dos pacientes na perspectiva dos médicos que atuam em uma maternidade. Método: Trata-se de um estudo transversal e quantitativo, com abordagem descritiva, realizado entre os meses de março e maio de 2016 com 124 médicos atuantes em uma maternidade pública de ensino do município de Fortaleza, Ceará, Brasil. As informações foram obtidas por meio do survey Hospital Survey on Patient Safety Culture (HSOPSC), Criado pela Agency for Healthcare Research and Quality (AHRQ), que avalia as percepções de profissionais em relação à segurança do paciente em 12 dimensões. Resultados: entre as 12 dimensões avaliadas, uma apresentou taxa de resposta positiva acima de 75\% (área fortalecida): aprendizado organizacional e melhoria contínua (80,4\%), e abaixo de 50\% (áreas com potencial de melhoria): as dimensões passagens de plantão/turno, transferências internas $(45,96 \%)$ e frequência de eventos notificados $(39,51 \%)$. Quanto a percepção geral sobre a segurança do paciente, necessita de melhorias na visão dos médicos. Conclusão: O estudo demonstrou a existência de uma cultura de segurança na instituição, mas com potencial de melhorias em algumas dimensões o que pode ser alcançado através do desenvolvimento de intervenções mais efetivas. Este tipo de investigação é útil pois serve como instrumento eficaz no planejamento, auxiliando os gestores nesta atividade.

Palavras-chave: Segurança do paciente. Cultura organizacional. Maternidades. Corpo Clínico.

\section{ABSTRACT}

Objective: To evaluate the safety culture of patients from the perspective of physicians who work in a maternity hospital. Method: This is a cross-sectional and quantitative study, with a descriptive approach, carried out between March and May 2016, with 124 physicians working in a public maternity hospital in the city of Fortaleza, Ceará, Brazil. The information was obtained through the Hospital on Patient Safety Culture (HSOPSC), Created by the Agency for Healthcare Research and Quality (AHRQ), which assesses the perceptions of professionals regarding patient safety in 12 dimensions. Results: among the twelve dimensions evaluated, one presented a positive response rate above $75 \%$ (strengthened area): organizational learning and continuous improvement $(80.4 \%$ ), and below 50\% (areas with potential for improvement): dimensions on-call transfers, internal transfers (45.96\%) and frequency of reported events (39.51\%). As for the general perception about patient safety, it needs improvements in doctors' eyesight. Conclusion: The study demonstrated the existence of a safety culture in the institution but with potential for improvement in some dimensions, which can be achieved through the development of more effective interventions. This type of research is useful as it serves as an effective planning tool, assisting managers in this activity.

Keywords: Patient safety. Organizational culture. Hospitals, maternity. Medical staff.

Autor correspondente: Ana Cláudia de Brito Passos, Rua Coronel Jaime Rolemberg, 950, Jardim Cearense, Ceará. CEP: 60712-175. Telefone: +55 85 3366-8293. E-mail: claudiab@ufc.br

Conflito de interesses: Não há qualquer conflito de interesses por parte de qualquer um dos autores.

Recebido em: 05 Mai 2019; Revisado em: 08 Fev 2020; Aceito em: 13 Ago 2020. 


\section{INTRODUÇÃO}

A segurança do paciente não é algo novo, mas a compreensão da magnitude do problema que envolve este tema é atual, sobretudo pelo número significativo de pacientes que são prejudicados durante os cuidados de saúde, como lesões permanentes, aumento do tempo de internação hospitalar, ou até mesmo a morte. Estudos apontam que os erros médicos são a terceira principal causa de morte nos Estados Unidos. No Reino Unido, recentes estimativas mostram que, em média, um incidente de dano ao paciente é relatado a cada 35 segundos. ${ }^{1}$

Melhorar a qualidade dos cuidados ofertados em maternidades, bem como a segurança dos pacientes e o gerenciamento de riscos, que envolvem o binômio mãe/feto-recém-nascido, particularmente em relação a eventos adversos, são questões importantes na área da saúde pública. ${ }^{2}$ Reduzir a mortalidade relacionada ao nascimento é hoje uma prioridade de saúde global, uma vez que a maioria das mortes e complicações que surgem durante a gravidez podem ser prevenidas e estão relacionadas com a qualidade da assistência prestada nos serviços de saúde. ${ }^{3,4} \mathrm{O}$ clima geral de segurança reduz a taxa de eventos obstétricos adversos, de modo que a avaliação e melhoria deste clima é o primeiro passo para reduzir o risco do paciente e criar uma cultura mais segura. ${ }^{4} \mathrm{~A}$ promoção da segurança eficaz dos pacientes, particularmente em maternidades, pode ser influenciada pela cultura organizacional, trabalho em equipe, comunicação aberta, feedbacks, respostas não punitivas a erros médicos e percepção comum das equipes sobre segurança. A fim de reduzir os erros obstétricos, é importante avaliar as fragilidades e os pontos fortes da cultura de segurança em maternidades. ${ }^{5}$

A cultura de segurança (CS) é definida como "o produto de valores, atitudes, percepções, competências e padrões de comportamentos individuais e de grupo, os quais determinam o compromisso, o estilo e proficiência da administração de uma organização de saúde com a gestão da segurança do paciente". ${ }^{6}$ No entanto, sabe-se que a CS não está relacionada apenas aos valores e atitudes compartilhados, mas que envolve padrões de comportamento e um esforço coletivo, onde todos os profissionais da instituição trabalham juntos para minimizar as falhas que prejudicam os pacientes. ${ }^{7}$

A Organização Mundial da Saúde (OMS) criou, em 2004, a Aliança Mundial para a Segurança do Paciente, onde uma das prioridades é a mensuração e avaliação rotineira da CS nas instituições de saúde, por meio de instrumentos validados. ${ }^{8}$ Por sua vez, o Ministério da Saúde brasileiro, através da criação do Programa Nacional de Segurança do Paciente, reconhece esta iniciativa estratégica da OMS, inferindo a importância de ser feita uma avaliação da CS, como condição fundamental para se introduzir mudanças nos comportamentos dos profissionais e instituições de saúde, e obter melhores níveis de segurança e de qualidade nos cuidados de saúde prestados aos pacientes. ${ }^{9}$

A avaliação da CS pode ser feita através do uso de questionários de autopreenchimento e por meio de técnicas de observação associadas a entrevistas. No entanto, de forma geral, a maioria das instituições de saúde avaliam a CS por meio de questionários como instrumentos de coleta de dados. Esses questionários são classificados como instrumento de avaliação psicométrica, fundamentam-se na combinação de dimensões da CS e são eficientes no processo de avaliação por serem anônimos e de baixo custo. ${ }^{6}$

Os dois questionários mais amplamente utilizados na avaliação da CS são a "Pesquisa sobre Segurança do Paciente em Hospitais (Hospital Survey on Patient Safety Culture (HSOPSC) criado pela Agency for Healthcare Research and Quality (AHRQ) e o Safety Attitudes Questionnaire (SAQ). Através das avaliações da CS do paciente nas instituições de saúde, potencialidades e fragilidades podem ser identificadas. ${ }^{6}$

No Brasil, a avaliação da CS em maternidades é relativamente recente, sendo importante realizar diagnóstico nestes contextos. Assim, verifica-se uma maior concentração de estudos de avaliação da CS em hospitais gerais, em grandes centros, com uma lacuna desse tipo de estudo em maternidades, particularmente envolvendo a equipe médica. ${ }^{10}$ Tais estudos serviriam para uma melhor compreensão sobre as atitudes e comportamentos desses profissionais bem como para o desenvolvimento da cultura de segurança. Desta forma, pretendeu-se, com este estudo, avaliar a CS em uma maternidade no nordeste do Brasil, na perspectiva dos profissionais médicos que ali atuam.

\section{MÉTODOS}

Trata-se de um estudo transversal e quantitativo, com abordagem descritiva, realizado entre os meses de março a maio de 2016, com 124 profissionais de saúde (médicos) que trabalham em uma maternidade pública do município de Fortaleza, Ceará, Brasil. A instituição nosocomial estudada desenvolve atividades de assistência, ensino, pesquisa e extensão, e integra o campus da Faculdade de Medicina, da Universidade Federal do Ceará (UFC).

Os critérios de inclusão utilizados neste estudo foram: ser médico da instituição; estar em atividades laborais ativamente; e aceitar os termos desta pesquisa com a assinatura do Termo de Consentimento Livre e Esclarecido (TCLE) e como critérios de exclusão, aqueles que estivessem afastados por licenças trabalhistas e férias.

O instrumento de coleta de dados foi o Inquérito Hospitalar sobre Cultura de Segurança do Paciente (Hospital Survey on Patient Safety Culture, HSOPSC), traduzido e validado para o Brasil, ${ }^{11}$ no qual se emprega a escala de Likert para examinar 12 dimensões de cultura de segurança do paciente, sete das quais estão relacionadas com as unidades/áreas do hospital, três estão relacionadas com o hospital e duas são variáveis de resultado. A escolha deste instrumento baseou-se na sua livre disponibilidade, no uso extenso em diferentes contextos culturais, e por suas propriedades psicométricas. 
O processo de coleta de dados ocorreu de duas formas com o intuito de conseguir o maior número de questionários respondidos: pelo formulário eletrônico disponível via internet e pessoalmente, com a pesquisadora in loco, ou seja, presente na maternidade em estudo, para abordagem dos profissionais que aceitassem participar da pesquisa.

Os dados foram inseridos em planilhas no programa Excel $\mathbb{R}$ for Windows ${ }^{\circledR}$, versão 2013 e, posteriormente, a frequência percentual de cada dimensão foi calculada e classificada, conforme preconiza a AHRQ em áreas frágeis de segurança do paciente, aquelas onde as dimensões com percentual de respostas positivas for inferior a $50 \%$ e, áreas fortalecidas da segurança do paciente (aquelas onde as dimensões com percentual de respostas positivas for igual ou maior que $75 \%)^{9}$

Aspectos éticos foram contemplados, seguindo-se a Resolução $n^{\circ}$ 466/2012, do Conselho Nacional de Saúde, obtendo-se, inclusive, a aprovação do estudo pelo Comitê de Ética em Pesquisa (CEP), da Universidade Federal do Ceará, por meio da Plataforma Brasil ( CAAE: 37872414.0.0000.5054).

\section{RESULTADOS}

A equipe médica da maternidade estava distribuída em diferentes unidades assistenciais, sendo que a maioria dos médicos trabalhava no centro cirúrgico $(49,19 \%)$, era do sexo feminino 57,26\%; a maior parte deles (46,77\%) possuía de 1 a 5 anos de trabalho na profissão, com regime de trabalho semanal menor que 40 horas semanais. Quanto ao vínculo com a instituição, 52,42\% dos médicos pertenciam à Empresa Brasileira de Serviços Hospitalares (EBSERH), $22,58 \%$ eram estatutários e $25 \%$ residentes. Em relação à formação profissional, $68,54 \%$ possuíam pós-graduação lacto sensu. Todos os profissionais tinham contato direto com os pacientes.

A Tabela 1 apresenta os percentuais de respostas positivas dos médicos que participaram da pesquisa, considerando cada dimensão estudada. Das doze dimensões analisadas, verificase que apenas a dimensão "aprendizagem organizacional e melhoria contínua" foi classificada como área fortalecida, uma vez que o percentual ficou acima de $75 \%$, seguindo as orientações dos autores do HSOPSC. Já em relação aos itens sobre "passagens de plantão/turno e transferências internas" e "frequência de eventos notificados", estes necessitam ser aprimorados, apresentando percentuais inferiores a 50\%.

A avaliação da CS em relação ao nível da unidade (Tabela 2), considerando os 12 itens componentes, verificou que o maior percentual de respostas positivas refere-se à pergunta que trata sobre o apoio das pessoas umas às outras; e, como ponto frágil, a questão que diz respeito ao fato de que o supervisor/chefe não dá atenção suficiente para os problemas relacionados a segurança do paciente que acontecem repetidamente.

Tabela 1. Percentual de respostas positivas dos profissionais médicos de acordo com o vínculo empregatício, conforme as dimensões da cultura de segurança do questionário HSOPSC. (a) $^{2}$

\begin{tabular}{|c|c|c|c|c|}
\hline DIMENSÕES & $\begin{array}{c}\text { EBSERH } \\
\begin{array}{c}(\mathrm{N}=65) \\
\%\end{array}\end{array}$ & $\begin{array}{c}\text { Estatutário } \\
\qquad \begin{array}{c}(\mathrm{N}=\mathbf{2 8}) \\
\%\end{array}\end{array}$ & $\begin{array}{c}\text { Residente } \\
\begin{array}{c}(\mathrm{N}=31) \\
\%\end{array}\end{array}$ & $\begin{array}{c}\text { Total } \\
(\mathrm{N}=124) \\
\%\end{array}$ \\
\hline \multicolumn{5}{|l|}{ Nível da unidade } \\
\hline Expectativas e ações de promoção de segurança dos supervisores/gerentes & 63,23 & 64,28 & 80,64 & 69,35 \\
\hline Aprendizado organizacional e melhoria contínua & 89,23 & 71,42 & 74,19 & 80,64 \\
\hline Trabalho em equipe dentro das unidades hospitalares & 64,61 & 57,14 & 77,41 & 66,12 \\
\hline Abertura da comunicação & 75,38 & 60,71 & 64,51 & 69,35 \\
\hline Retorno das informações e comunicação sobre os erros & 59,92 & 50,00 & 64,51 & 57,25 \\
\hline Resposta não punitiva aos erros & 73,86 & 71,42 & 64,51 & 70,96 \\
\hline Adequação de Profissionais & 69,23 & 75,00 & 67,74 & 70,16 \\
\hline \multicolumn{5}{|l|}{ Nível da organização hospitalar } \\
\hline Apoio da gestão hospitalar para segurança do paciente & 80,00 & 57,14 & 80,64 & 73,38 \\
\hline Trabalho em equipe entre as unidades hospitalares & 64,61 & 57,14 & 77,41 & 66,12 \\
\hline Passagens de plantão/turno e transferências internas & 46,15 & 46,42 & 51.61 & 45,96 \\
\hline \multicolumn{5}{|l|}{ Medidas de resultados } \\
\hline Percepção geral de segurança do paciente & 55,38 & 64,28 & 64,51 & 54,03 \\
\hline Frequência de eventos notificados & 36,92 & 46,42 & 38,70 & 39,51 \\
\hline
\end{tabular}

(a)Hospital Survey on Patient Safety Culture - avaliação da cultura de segurança do paciente nos Serviços de Saúde/2016. 
Tabela 2. Respostas positivas às dimensões da cultura de segurança em nível da unidade do questionário HSOPSC ${ }^{(a)}$ e seus respectivos itens componentes entre médicos de uma maternidade no nordeste Brasileiro.

\begin{tabular}{|c|c|}
\hline Dimensões e itens componentes & $\begin{array}{l}\text { Respostas Positivas } \\
\%\end{array}$ \\
\hline \multicolumn{2}{|l|}{ 1. Expectativas do supervisor/chefe e ações promotoras de segurança } \\
\hline $\begin{array}{l}\text { B1. O meu supervisor/chefe elogia quando vê um trabalho realizado de acordo com os procedimentos } \\
\text { estabelecidos de segurança do paciente }\end{array}$ & 63,70 \\
\hline $\begin{array}{l}\text { B2. O meu supervisor/chefe realmente leva em consideração as sugestões dos profissionais para a } \\
\text { melhoria da segurança do paciente }\end{array}$ & 69,35 \\
\hline $\begin{array}{l}\text { B3. Sempre que a pressão aumenta, o meu supervisor/chefe quer que trabalhemos mais rápido, mesmo que } \\
\text { isso signifique "pular etapas" }\end{array}$ & 40,32 \\
\hline $\begin{array}{l}\text { B4. O meu supervisor/chefe não dá atenção suficiente aos problemas de segurança do paciente que } \\
\text { acontecem repetidamente }\end{array}$ & 29,03 \\
\hline \multicolumn{2}{|l|}{ 2. Aprendizado organizacional e melhoria contínua } \\
\hline A6. Estamos ativamente fazendo coisas para melhorar a segurança do paciente & 80,64 \\
\hline A9. Erros, enganos ou falhas têm levado a mudanças positivas por aqui & 71,77 \\
\hline A13. Após implementarmos mudanças para melhorar a segurança do paciente, avaliamos a efetividade & 56,45 \\
\hline \multicolumn{2}{|l|}{ 3. Trabalho em equipe dentro da unidade } \\
\hline A1. Nesta unidade as pessoas apoiam umas as outras & 83,06 \\
\hline $\begin{array}{l}\text { A3. Quando há muito trabalho a ser feito rapidamente, trabalhamos juntos em equipe para concluí-lo } \\
\text { devidamente }\end{array}$ & 75,80 \\
\hline A4. Nesta unidade as pessoas se tratam com respeito & 61,29 \\
\hline A11. Quando uma área/unidade de trabalho fica sobrecarregada, as outras ajudam & 55,68 \\
\hline \multicolumn{2}{|l|}{ 4. Abertura da comunicação } \\
\hline $\begin{array}{l}\text { C2. Os profissionais têm liberdade para dizer ao ver algo que pode afetar negativamente o cuidado do } \\
\text { paciente }\end{array}$ & 69,65 \\
\hline C4. Os profissionais sentem-se à vontade para questionar as decisões ou ações de seus superiores & 43,54 \\
\hline C6. Os profissionais têm receio de perguntar, quando algo parece não estar certo & 30,64 \\
\hline \multicolumn{2}{|l|}{ 5. Retorno da informação e comunicação sobre os erros } \\
\hline C1. Nós recebemos informação sobre mudanças implementadas a partir de relatórios de eventos & 49,19 \\
\hline C3. Nós somos informados sobre os erros que acontecem nesta unidade & 38,70 \\
\hline C5. Nesta unidade, discutimos meios de prevenir erros para que eles não aconteçam novamente & 57,25 \\
\hline \multicolumn{2}{|l|}{ 6. Resposta não punitiva aos erros } \\
\hline A8. Os profissionais consideram que seus erros, enganos ou falhas podem ser usados contra eles & 70,97 \\
\hline A12R. Quando um evento é relatado, parece que o foco recai sobre a pessoa e não sobre o problema & 49,19 \\
\hline $\begin{array}{l}\text { A16. Os profissionais se preocupam que seus erros, enganos ou falhas sejam registrados em suas fichas } \\
\text { funcionais }\end{array}$ & 55,64 \\
\hline \multicolumn{2}{|l|}{ 7. Adequação de pessoal } \\
\hline A2. Temos profissionais suficientes para dar conta da carga de trabalho & 57,26 \\
\hline $\begin{array}{l}\text { A5. Nesta unidade, os profissionais trabalham mais horas do que seria o melhor para o cuidado do } \\
\text { paciente }\end{array}$ & 45,97 \\
\hline $\begin{array}{l}\text { A7. Utilizamos mais profissionais temporários/terceirizados do que seria desejável para o cuidado do } \\
\text { paciente }\end{array}$ & 36,29 \\
\hline A14. Nós trabalhamos em "situação de crise", tentando fazer muito e muito rápido & 70,16 \\
\hline
\end{tabular}

(a)Hospital Survey on Patient Safety Culture - avaliação da cultura de segurança do paciente nos Serviços de Saúde/2016. 
Analisando, mais detalhadamente, cada dimensão como foco para a unidade de trabalho, e seus itens componentes, foi observado que os que obtiveram menor percentual de respostas positivas foram aqueles relativos ao "retorno da informação e comunicação sobre os erros".

Destaca-se, ainda, na análise das dimensões da CS, no item "aprendizado organizacional e melhoria contínua", que a melhor avaliação considerada foi a que menciona que "estamos ativamente fazendo coisas para melhorar a segurança do paciente" (80,64\%). Entretanto, um aspecto a ser questionado é que o percentual obtido $(56,45 \%)$ no item "após implementarmos mudanças para melhorar a segurança do paciente, avaliamos a efetividade", demonstrou que o processo precisa ser revisto.

Na dimensão "respostas não punitivas aos erros", pode-se destacar um percentual de $70,97 \%$ da equipe médica considerando que seus erros, enganos ou falhas podem ser usados contra eles. Além disso, foi observado percentual significativo $(49,19 \%)$ de respostas denotando a ideia que a notificação dos eventos tem o foco sobre a pessoa, e não sobre o problema.

Do ponto de vista da "organização hospitalar", destacou-se a dimensão da CS “apoio da gestão hospitalar para segurança do paciente", com 73,39\% de respostas positivas. Como ponto fraco, foi identificado o item "a direção do hospital só parece interessada na segurança do paciente quando ocorre algum evento adverso" (32,25\%).

$\mathrm{Na}$ dimensão de "passagens de plantão ou de turno/ transferências", todos os itens são pontos a ser melhorados na instituição, destacando-se, como aquele de menor percentual $(33,06 \%)$, o item que afirma que as mudanças de plantão ou de turno são problemáticas para os pacientes.

Quanto às medidas de resultados, verificou-se que, em relação à percepção geral da segurança do paciente, o resultado mais crítico foi o item que afirma ser "apenas por acaso que erros, enganos ou falhas não acontecem por aqui”, com 36,29\% de respostas positivas. Quanto à frequência de eventos notificados, todos os itens obtiveram percentuais abaixo de 50\%.

No tocante à avaliação do grau de segurança do paciente (Figura 1), 64,62\% dos participantes pertencentes ao quadro da Empresa Brasileira de Serviços Hospitalares (EBSERH), consideraram esta avaliação como "muito boa". Por sua vez, quanto ao número de notificações feitas nos últimos 12 meses, de acordo com o vínculo institucional, destacam-se os médicos da EBSERH (58,82\%), com mais de uma notificação realizada.

Figura 1. Avaliação do grau de segurança do paciente, na perspectiva dos médicos de uma maternidade do nordeste brasileiro.

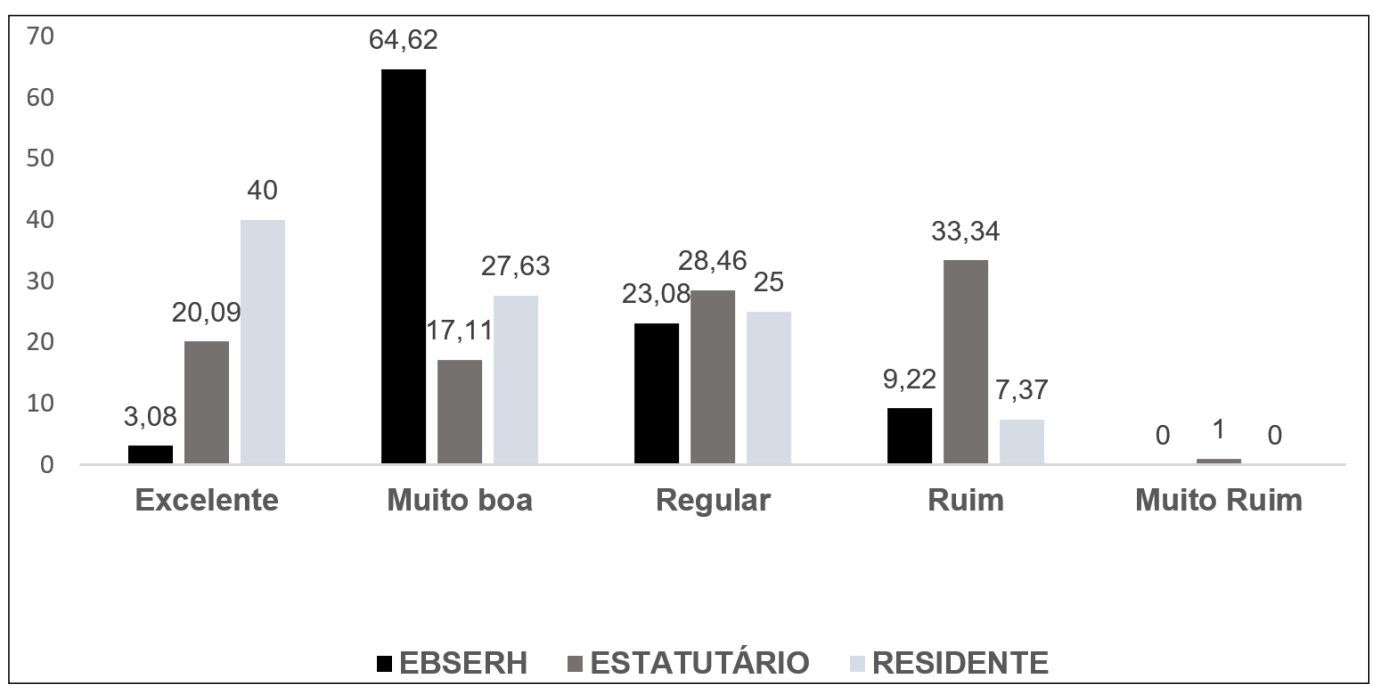

\section{DISCUSSÃO}

Ao se analisar a maioria das dimensões apontadas pelo instrumento HSOPSC verificou-se, pelos resultados apresentados, que já existe uma cultura de segurança do paciente (CSP), que pode ser considerada como "forte", na instituição estudada. Entretanto, foram identificadas, também, dimensões com resultados críticos e que precisam ser melhoradas, a saber, aspectos envolvendo as passagens de plantão/turno da equipe médica e transferências de pacientes de uma unidade para outra, bem como a frequência de eventos notificados.
Sobre o perfil dos participantes do nosso estudo, a maior parte dos médicos pertencia à EBSERH, pois a empresa foi criada para repor a força de trabalho terceirizada, antes existente, e a carência da instituição, estando em consonância com estratégias governamentais e nacional, no contexto da implementação da gestão nosocomial. A diferença entre os vínculos pode gerar impacto sobre a cultura de segurança, pois os estatutários estão há mais tempo e trazem consigo a cultura preexistente, os novos (EBSERH), dependendo do tempo de serviço na instituição, podem ainda ter opiniões contaminadas pela cultura dos seus locais de origem. Por sua vez, quanto aos 
residentes, é importante inferir que, por estarem em processo de formação/conhecimento, suas percepções acerca da CSP podem constituir reflexões da formação acadêmica recebida com foco para a segurança do paciente. De fato, em várias matrizes curriculares das residências na área de saúde, já se contemplam aspectos relacionados à segurança do paciente, o que é bem positivo.

Problemas de comunicação foram detectados entre os médicos nos momentos de 'passagem de plantão/turno'. Dos Santos et al., (2018) $)^{12}$ relatam que problemas de comunicação têm sido associados com a diminuição de qualidade de cuidados e com o número de mortes por erros médicos que poderiam ser evitados.

Sobre a frequência de eventos notificados, sabe-se que a CSP é um fator importante para reduzir a ocorrência desses eventos e para melhorar a segurança do paciente. Na verdade, o aprendizado organizacional ocorre a partir das falhas, prevenindo novos incidentes relacionados à assistência à saúde. ${ }^{13,14}$ Nesse sentido, nossos achados foram capazes de expressar a existência de uma fragilidade, na instituição, no tocante a frequência das notificações, o que sugere apreensão dos médicos ao vivenciarem ou reportarem algum incidente, possivelmente por receio que tal conduta possa ser usada contra eles, o que induz a subnotificação.

Estudo de Siman, Cunha e Brito (2017), ${ }^{15}$ realizado em um hospital de ensino sobre a prática de notificação de eventos adversos, verificou que a existência de uma cultura punitiva e lacunas no conhecimento são barreiras na prática efetiva das notificações de eventos adversos. Ressalta ainda, que é importante investir na disseminação da cultura de segurança para que melhorias possam acontecer neste contexto.

A dimensão de trabalho em equipe, no âmbito das unidades, foi a que recebeu melhor avaliação, assim como a aprendizagem organizacional e a melhoria contínua, que obteve o segundo melhor resultado. De fato, o trabalho em equipe é fundamental para a segurança do paciente, sendo visto pelos profissionais que participaram desta pesquisa de forma positiva e benéfica, em concordância com resultados também encontrados em outros estudos. Sabe-se que o trabalho em equipe requer comunicação efetiva, prática colaborativa, empatia e apoio, aspectos fundamentais para melhoria da segurança do paciente. ${ }^{5,15-19}$

Quanto ao aprendizado organizacional e a melhoria contínua, os pontos fortes evidenciados foram: a unidade de trabalho ser ativa, em relação às ações de segurança do paciente e, o aprendizado, a partir dos erros cometidos. Nesse contexto, vários estudos corroboram com estes resultados, ${ }^{19-21}$ demonstrando que nossa avaliação, além de ter sido capaz de identificar as potencialidades da instituição, as identificou de forma precisa e consonante com outros estudos de abordagem semelhante.

Em relação ao apoio da gestão hospitalar para a segurança do paciente verificou-se uma avaliação positiva nos itens relativos aos elogios feitos pelo supervisor aos profissionais quando realizavam ações para segurança do paciente, e pelo aproveitamento das sugestões feitas por esses mesmos profissionais. Resultados semelhantes foram encontrados em outros estudos. ${ }^{15,22}$ Um aspecto frágil e preocupante identificado nessa dimensão, foi o relato de que o supervisor/ chefe não disponibilizava atenção suficiente aos problemas de segurança do paciente que aconteciam repetidamente.

Na dimensão 'respostas não punitivas ao erro', verificou-se como único ponto fraco a punição recair sobre a pessoa quando um erro é cometido. Na verdade, o que se deve atentar é que o erro seja considerado como algo que conduza à aprendizagem organizacional, sendo utilizado como instrumento de melhoria contínua para a segurança do paciente. Quando este fato acontece, o erro fica restrito ao problema ocorrido, e a tendência é que o profissional, diante desta abordagem, temendo punições esconda suas falhas, além de vivenciar sentimentos como frustração ou vergonha. ${ }^{23,24}$ Desse modo, a organização fica impossibilitada de avaliar essas falhas, corrigi-las e evitá-las.

Neste aspecto, o estudo de Silva, Pedrosa, Leça e Silva, ${ }^{25}$ mostrou que a existência de respostas punitivas contribuiu para a não notificação de incidentes, causando problemas na implementação de medidas corretivas, uma vez que poderá levar os profissionais a negligenciar informações importantes sobre os erros, limitando a sua análise.

Para o conjunto de itens da dimensão "percepção geral da segurança do paciente em sua unidade de trabalho", o resultado foi positivo. Entretanto, o percentual de respostas negativas relacionado ao item "a ocorrência de erros mais graves não acontece apenas por acaso" chama atenção para a fragilidade da cultura de segurança. Salienta-se a necessidade de melhorias em relação ao assunto, com maior planejamento e uso de protocolos para que não se dependa do acaso, mas sim de ações eficientes e conscientes. Sabe-se que a percepção da segurança requer um amadurecimento dos profissionais de saúde, não envolvendo simplesmente mudanças de regras, rotinas ou procedimentos, mas também mudanças culturais e de comportamento, o que dificulta a melhoria nesse âmbito, ${ }^{15}$ mesmo em países desenvolvidos e pioneiros na abordagem e contexto envolvendo a segurança do paciente.

Neste estudo, a dimensão "abertura da comunicação", foi vista de forma positiva pelos participantes da pesquisa, fator relevante para o bom desenvolvimento da CSP. O ponto fraco identificado foi a ausência de recebimento de informações sobre mudanças implementadas a partir dos relatórios de eventos. A comunicação é uma área importante para a segurança do paciente, sendo fundamental que os profissionais tenham conhecimento de todos os processos, mudanças, problemas ocorridos em sua unidade, para que possam agir de forma coerente em relação à segurança do paciente. ${ }^{12}$ A promoção efetiva da segurança do paciente, particularmente em maternidades, pode ser influenciada pela cultura organizacional, trabalho em equipe, comunicação aberta, feedbacks, respostas não punitivas a erros, e percepção das equipes sobre a segurança do paciente. 
A frequência de eventos notificados recebeu a pior avaliação, demonstrando que muitos erros podem passar despercebidos, prejudicando a segurança do paciente no hospital. Estudo realizado em hospital de ensino público, ${ }^{16}$ encontrou resultado semelhante ao nosso, evidenciando que a baixa notificação de eventos pode ser relacionada com as respostas punitivas decorrentes das falhas profissionais.

Como limitações deste estudo, tem-se a ausência de análise estatística inferencial, bem como a escassez de estudos realizados em maternidades com essa abordagem, e também o fato de que a maioria dos trabalhos avalia a percepção sobre a CS não apenas dos médicos, mas de outros profissionais de saúde, dificultando fazer comparações e análises mais detalhadas.

\section{CONCLUSÃO}

A avaliação da cultura de segurança nos serviços de saúde é o primeiro passo para a busca de melhorias na qualidade e na segurança nos cuidados de saúde, fornecendo informações

\section{REFERÊNCIAS}

1. World Health Organization. Patient safety: making health care safer. Geneva: World Health Organization; 2017 [acesso em: 10 jun 2020]. Disponível em: https://apps.who.int/iris/ handle/10665/255507

2. Batista AC. Cultura de segurança do paciente na perspectiva de profissionais da enfermagem obstétrica e neonatal [dissertação]. Florianópolis: Universidade Federal de Santa Catarina; 2015.

3.Calderon IM, Cecatti JG, Vega CE. Intervenções benéficas no pré-natal para prevenção da mortalidade maternal. Rev Bras Ginecol Obstet. 2006;28(5):310-15.

4. Raftopoulos V, Savva N, Papadopoulou M. Safety culture in the Maternity Units: a censos survey using the Safety Attitudes Questionnaire. BMC Health Serv Res. 2011;11(238):1-10.

5. Akbari N, Malek M, Ebrahimi P, Haghan H, Aazami S. Safety culture in the maternity unit of hospitals in Ilam province, Iran: a census survey using HSOPSC tool. Pan Afr Med J. 2017;27:268.

6. Nieva VF, Sorra J. Safety culture assessment: a tool for improving patient safety in healthcare organizations. Qual Saf Health Care. 2003; 12(Suppl 2):17-23

7. Sousa P, Mendes W. Segurança do paciente: criando organizações seguras. $2^{\mathrm{a}}$ ed. Rio de Janeiro: EAD/ENSP; 2014. 208 p.

8. Brasil. Ministério da Saúde. Documento de referência para o Programa Nacional de Segurança do Paciente. Brasília: Ministério da Saúde; 2014. 40 p.

9. Brasil. Ministério da Saúde. Portaria № 529, de $1^{\circ}$ de abril de 2013. Institui o Programa Nacional de Segurança do Paciente (PNSP). Brasília: Ministério da Saúde; 2013. [acesso em 10 jun 2020]. Disponível em: https://bvsms.saude.gov.br/bvs/saudelegis/gm/2013/ prt0529_01_04_2013.html

10. Reis CT, Paiva SG, Sousa P. The patient safety culture: a para os líderes organizacionais em relação aos pontos fortes (potencialidades) e fragilidades, em diferentes áreas e escopos, que poderão subsidiar a tomada de decisões nesta área.

Nossos achados demonstraram a existência de uma CS na instituição estudada, nos principais aspectos abordados no questionário utilizado, com potencial de melhoria nas dimensões de "passagens de plantão/turno e transferências internas", e de "frequência de eventos notificados".

Verdadeiramente, avaliar a CS é uma ferramenta essencial para a melhoria da qualidade nos cuidados prestados aos pacientes e isso representa um esforço coletivo de profissionais, gestores, pacientes, academia e a sociedade em geral. Sabe-se que uma mudança de cultura nunca será algo fácil, entretanto, é factível. Por essa razão, a adoção da cultura de segurança é uma estratégia a ser desenvolvida ao longo de um período de tempo, com treinamento e capacitação constante dos profissionais, constituindo-se um processo que envolve educação e mudança de hábito.

systematic review by characteristics of Hospital Survey on Patient Safety Culture dimensions. Int J Qual Health Care. 2018;30(9):66077.

11. Reis CT, Laguardia J, Martins M. Adaptação transcultural da versão brasileira do Hospital Survey on Patient Safety Culture: etapa inicial. Cad Saude Pública. 2012;28(11):2199-210.

12. Santos MC, Grilo A, Andrade G, Guimarães T, Gomes A. Comunicação em saúde e a segurança do doente: problemas e desafios. Rev Port Saúde Pública. 2010; Vol Temat(10):47-57.

13. Souza VS, Kawamoto AM, Oliveira JL, Tonini NS, Fernandes LM, Nicola AL. Erros e eventos adversos: a interface com a cultura de segurança dos profissionais de saúde. Cogitare Enferm. $2015 ; 20(3): 474-81$.

14. Siman AG, Cunha SG, Brito MJ. A prática de notificação de eventos adversos em um hospital de ensino. Rev Esc Enferm USP. 2017;51; e03243

15. Mello JF, Barbosa SF. Cultura de segurança do paciente em terapia intensiva: recomendações da enfermagem. Texto Contexto Enferm. 2013;22(4):1124-33.

16. Gama ZA, Oliveira AC, Hernández, PJ. Cultura de seguridad del paciente y factores asociados en una red de hospitales públicos españoles. Cad Saúde Pública. 2013;29(2):283-93.

17. Fernandes AM, Queirós PJ. Cultura de Segurança do Doente percecionada por enfermeiros em hospitais distritais portugueses. Rev Enf Ref. 2011;serIII(4):37-48.

18. Wang M, Tao H. How does patient safety culture in the surgical departments compare to the rest of the county hospitals in Xiaogan City of China? Int J Environ Res Public Health . 2017;14(10):1123.

19. Wami SD, Demssie AF, Wassie MM, Ahmed AN. Patient safety culture and associated factors: A quantitative and qualitative study 
of healthcare workers' view in Jimma zone Hospitals, Southwest Ethiopia. BMC Health Serv Res. 2016;16:495.

20. El-Jardali F, Sheikh F, Garcia NA, Jamal D, Abdo A. Patient safety culture in a large teaching hospital in Riyadh: baseline assessment, comparative analysis and opportunities for improvement. BMC Health Serv Res. 2014;14(2014):122

21. Minuzzi AP, Salum NC, Locks MO. Avaliação da cultura de segurança do paciente em terapia intensiva na perspectiva da equipe de saúde. Texto \& Contexto - enferm. 2016;25(2): e1610015

22. Fujita S, Seto K, Ito S, Wu Y, Huang CC, Hasegawa T. The characteristics of patient safety culture in Japan, Taiwan and the United States. BMC Health Ser Res. 2013;13:20

23. Françolin L, Gabriel CS, Bernardes A, Silva AE, Brito MF, Machado JP. Gerenciamento da segurança do paciente sob a ótica dos enfermeiros. Rev Esc Enferm USP. 2015;49(2):277-83

24. Silva MV, Carvalho PM. Cultura de segurança do paciente: atitudes dos profissionais de enfermagem de um serviço de prontoatendimento. R. Interd. 2016;9(1):1-12.

25. Silva EM, Pedrosa DL, Leça AP, Silva DM. Percepção dos profissionais de saúde sobre a cultura de segurança do doente pediátrico. Rev Enf. Ref. 2016;serIV(9):87-95.

\section{Como citar:}

Barillas CC, Passos AC, Alencar CA Júnior, Lopes EM, Neri ED, Fonteles MM, et al. Cultura de segurança em uma maternidade pública de ensino: conhecer para intervir. Rev Med UFC. 2021;61(1):1-8. 